\title{
Hydraulic Analysis of the Wendelstein 7-X Cooling loops
}

\author{
M.Smirnow ${ }^{\mathrm{a}}$, G.Orozco ${ }^{\mathrm{a}}$, J.Boscary ${ }^{\mathrm{a}}$, A.Peacock ${ }^{\mathrm{b}}$ \\ ${ }^{a}$ Max-Planck-Institut für Plasmaphysik, EURATOM-Assoziation, Boltzmannstr. 2, 85748 Garching, Deutschland \\ ${ }^{b}$ European Commission c/o Max-Planck-Institut für Plasmaphysik, EURATOM Association, Boltzmannstr. 2, 85748 Garching, Deutschland
}

\begin{abstract}
Actively water cooled in vessel components (IVC) are required for the long pulse operation of the stellarator Wendelstein 7-X (W7-X). In total, the cooling pipes have a length of about $4.5 \mathrm{~km}$, supplying the coolant via 304 cooling circuits for the IVC. Within each cooling loop, the IVC are organized mostly in parallel. A homogeneous flow through all branches or at least the minimum specified flow in all of the branches of a circuit is crucial for the IVC to withstand the loading conditions. A detailed hydraulic simulation model of the W7-X cooling loops was built with the commercial code Flowmaster, which is a 1-D computational fluid dynamics software. In order to handle the huge amount of pipe-work data that had to be modelled, a pre- and post-processing macro was developed to transfer the 3D Catia V5 CAD model to the 1-D piping model. Within this model, the hydraulic characteristics of different types of first wall components were simulated, and compared with their pressure drop measurements. As a result of this work, the need for optimization of some cooling loops has been identified and feasible modified solutions were selected.
\end{abstract}

Keywords: Wendelstein 7-X, stellarator plasma facing components, first wall, computational fluid dynamics

\section{Introduction}

Actively water cooled in vessel components (IVC) [1][2] are mandatory for the steady-state operation of Wendelstein 7-X (W7-X), with 10MW input power over a pulse length of up to $30 \mathrm{~min}$ and reaching peak input power loads up to 20MW for 10s. 304 cooling loops with 22 baseline types and 106 variants supply about 1500 in vessel components, mostly organized in parallel and sometimes in serial. 80 ports of the machine distribute the required water to the different IVC [3]. The hydraulic analysis presented here focused on the cooling loops of the baffles and the first wall components: heat shields and panels. The cooling parameters of the different type of plasma facing components depends on the loading conditions listed in Table 1.

Table 1: List of investigated IVC's for the hydraulic study

\begin{tabular}{ccccc}
\hline \hline IVC type & Count & Heatload & inlet Pressure & Velocity \\
\hline & $\#$ & {$\left[\mathrm{~kW} / \mathrm{m}^{2}\right]$} & {$[\mathrm{MPa}]$} & {$[\mathrm{m} / \mathrm{s}]$} \\
\hline Baffle & 170 & 250 & 2.5 & 6 \\
Heat shield & 162 & $150-250$ & 2.5 & 6 \\
Panels & 320 & 100 & 2.5 & 2 \\
\hline \hline
\end{tabular}

Email address: michael.smirnow@ipp.mpg.de (M.Smirnow)
The baffle and heat shields are based on the same technology [4]. The function of the baffle, which are parts of the divertor system, is to improve the pumping efficiency. The heat shields and panels [5] protect the plasma vessel wall, the pipes of the cooling loops, and integrated diagnostics with their instrumentation.

\section{Purpose of the hydraulic analysis}

The purposes of the hydraulic study were:

- The calculation of the pressure drop of the individual components and the comparison with the measured values. An agreement within $\pm 15 \%$ has been specified.

- The calculation of the pressure drop of the individual cooling loops to confirm that the design meets the requirement of a total pressure drop lower than 10 bar for an axial velocity given in Table 1 for each component.

- In case of parallel arrangement, the calculation of the velocity distribution to ensure an axial velocity given in Table 1 in each of the branches.

August 13, 2013 
- In case of the use of flexible hoses in the circuit, the calculation ensures that the axial velocity in the hoses is below $3 \mathrm{~m} / \mathrm{s}$.

\section{Theory description of the hydraulic modelling}

The cross section of the pipes used for the cooling circuits is circular. In the case of a constant cross-section, the pressure drop is expressed as the sum of the friction coefficients according to the Darcy-Weissbach equation.

$$
\Delta P=\lambda \cdot \frac{L}{D} \cdot \frac{\rho \cdot u^{2}}{2}=k \cdot Q^{2}
$$

with

$$
k=\sum\left(\zeta_{i}\right) \cdot \frac{\rho}{2 \cdot S^{2}}
$$

Where $\lambda$ the pressure drop coefficient, $\mathrm{D}$ the hydraulic diameter, $\mathrm{L}$ the length of the pipe, $\rho$ the fluids density, $\mathrm{u}$ the coolant velocity, $\mathrm{S}$ the cross-sectional wetted area and $\mathrm{Q}$ the volumetric flow rate.

Thus the hydraulic characteristics of a component or pipe is the $\mathrm{k}$-Value, which is the slope of the measured curve $\Delta P$ vs. $Q^{2}$. The sum of friction factors $\zeta$ is a function of the wall roughness and Reynolds number.

\section{Hydraulic modelling of a baffle module}

The cooling structure of a baffle is a meander made of stainless steel 1.4441. The geometry of the pipe is $12 \times 1 \mathrm{~mm}$ and the roughness is $20 \mu \mathrm{m}$ based on experimental measurements. The cooling structure is build up from a combination of straight pipes, bends and miter joints (Fig.1). The commercial code Flowmaster ${ }^{\mathrm{TM}}[6]$

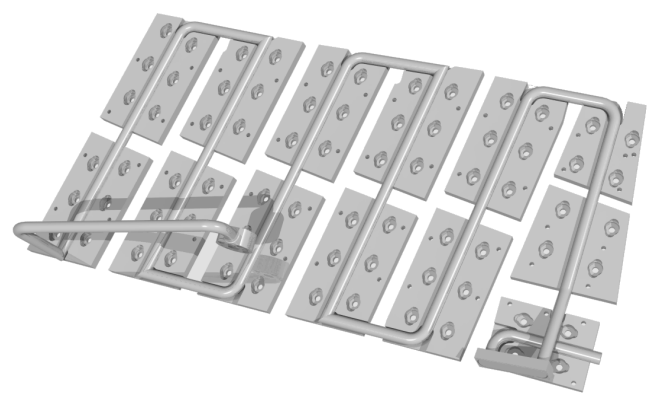

Figure 1: Rear view to the Baffle Module $2 \mathrm{H}$ cooling structure.

has a large catalogue of already configured hydraulic parts as pipes, bends, miter joints and junctions. In order to model the large amount of different components, the $3 \mathrm{D}$ CAD models are derived from CATIA ${ }^{\mathrm{TM}}$ and translated into a 1D Flowmaster model as a series of straight and bent pipes with miter joints (Fig.2) with VBA-Macros.

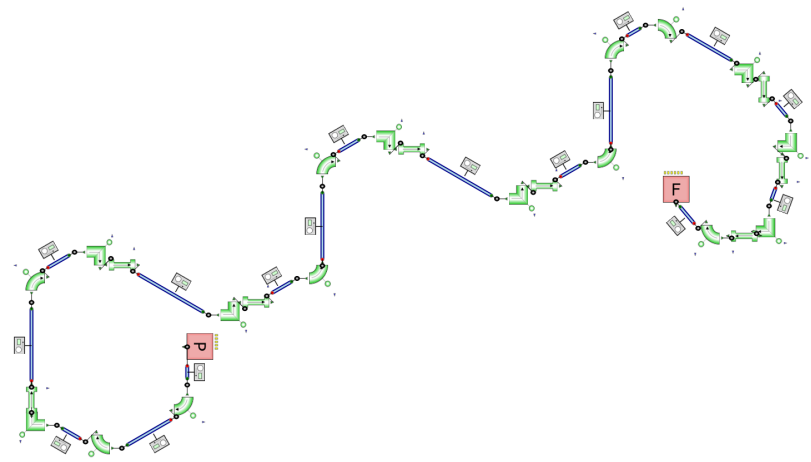

Figure 2: Description of the Baffle Module 2H cooling loops for the hydraulic study in Flowmaster ${ }^{\mathrm{TM}}$

This Macro uses the application programming interface (API) of CATIA and Flowmaster and is controlled from inside the Microsoft Excel environment. Fig.3 explains the workflow of the Macro, the information paths are indicated with arrows. The cooling pipe structure in the 3D-CAD Model is measured manually with CATIA tools. The list of measurements is imported into Excel, building the basis for the automated pipe network construction (FMDNA) in Flowmaster ${ }^{\mathrm{TM}}$. Once the network is build, the Macro sets the boundary conditions for the simulation and starts the solver for a range of inlet flow rates and provides the pressure drop for each inlet flow value as a result. The simulated component's pressure drop vs. flow characteristic curve is stored in the Flowmaster SQL-Database. Then the measurements are imported and compared to the calculated characteristics and a report is generated.

\section{Hydraulic modelling of a cooling loop}

The cooling loop network model in Flowmaster ${ }^{\mathrm{TM}}$ is build up by components loaded from the SQL Database and connected through pipes with junction elements. Fig. 4 shows the cooling loop of panels arranged in parallel. The geometry of the cooling channel of the panels is more complex than the baffles and heat shields. The panels are made from a sandwich of two stainless steel sheets. The two sheets are laser-welded together to form a meander of cooling channels. Along the circumference a vacuum tight weld seals the sandwich [1]. The pressure drop characteristics were not simulated and only the measured k-values were used in the calculation [7]. The parts which connects the panels are 


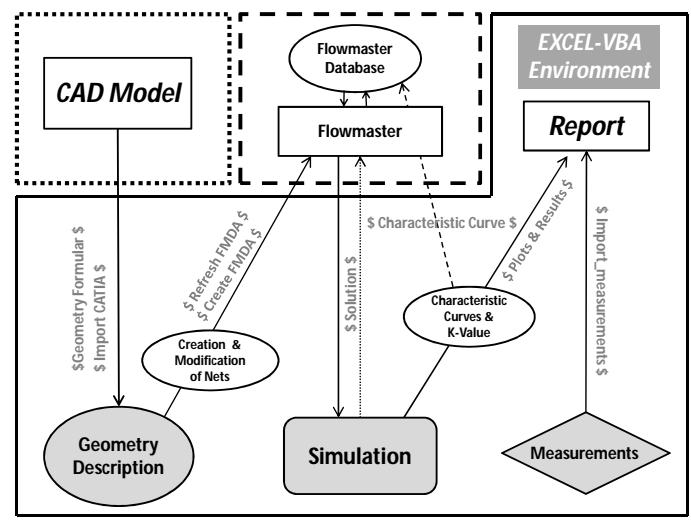

Figure 3: Organigramm of the VBA-Macro workflow

straight pipes, flexible hoses, orifices and junctions. The corresponding Flowmaster ${ }^{\mathrm{TM}}$ model is shown in Fig.5. At the pressure node - marked as P in the Fig. 5 - an inlet pressure of $2.5 \mathrm{MPa}$ and a nominal flow of $1.8 \mathrm{~kg} / \mathrm{s}$ at $20^{\circ} \mathrm{C}$ - marked as $\mathrm{F}$ in the Fig.5 - are applied. The cooling loop consists of 8 components arranged in parallel. Measured pressure drop showed that one of the component has a value about $70 \%$ lower than the average of the other components. The purpose of the calculation was to define the characteristics of the needed orifice in this branch and to check that the required velocities are reached in the other branches.

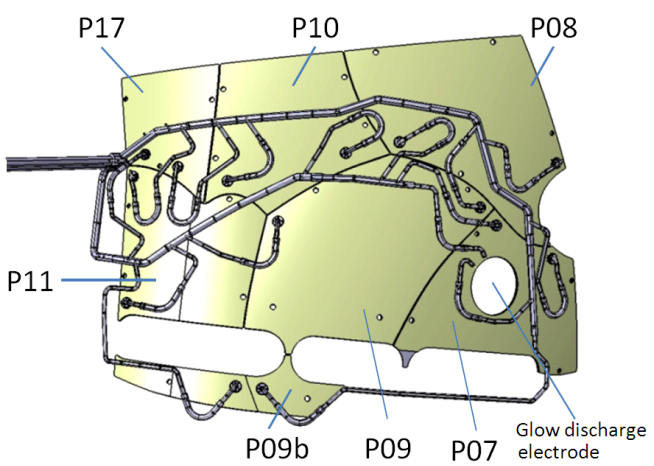

Figure 4: Cooling loop network $3 \mathrm{c}$ as designed in CATIA

3-D hydrodynamic Ansys-CFX ${ }^{\mathrm{TM}}$ calculations were performed on various types of orifices with different diameter. The selected geometry, shown in Fig.6, has the advantage of a larger diameter, which reduces the possible risk of blocking during operation. The pressure drop characteristics corresponds to an orifice of a 3.4 $\mathrm{mm}$ diameter. A prototype of this orifice was built and measured. The measured pressure drop characteristic of

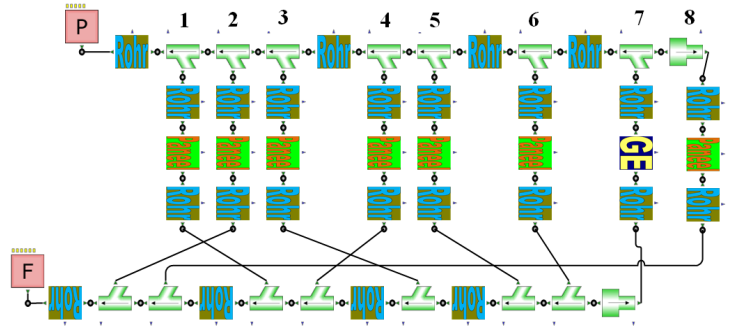

Figure 5: Cooling loop network $3 \mathrm{c}$ in Flowmaster ${ }^{\mathrm{TM}}$

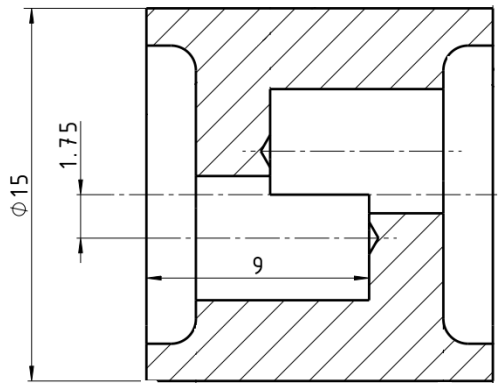

Figure 6: Crosssection of the eccentric orifice branches.

this orifice is then used in the following simulations.

\section{Analysis of the simulations}

Fig.7 shows an overall comparison between the measured $\mathrm{k}$-values and the calculated $\mathrm{k}$-values of all the variants of baffles and heat shields. In a $\mathrm{k}$-value range from $10-17\left(0.1 * \mathrm{MPa} /\left(\mathrm{m}^{3} / \mathrm{s}\right)^{2}\right)$ there is a good correlation between measurements and simulations. Above this range, there is a tendency to overestimate the measurement by about $15 \%$ especially for heat shields.

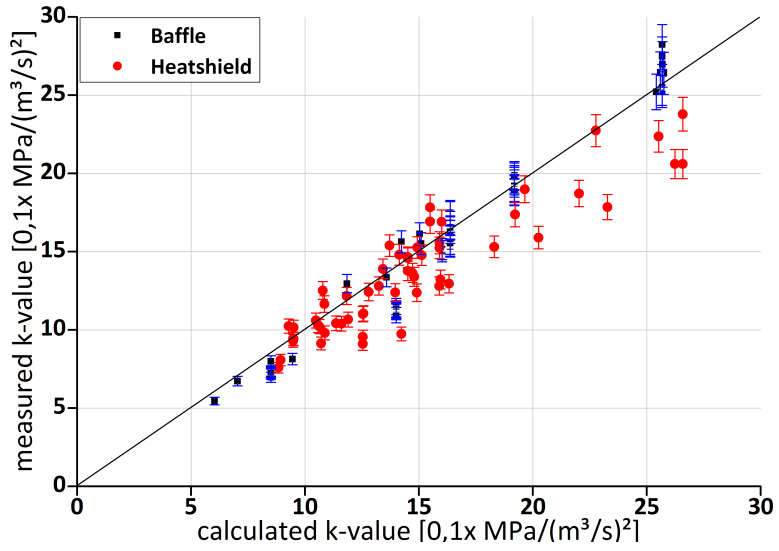

Figure 7: Overall comparison between measured and simulated kvalue for the Baffle and Heat shield Modules 
W7-X is a five-fold symmetry machine featuring 10 half-modules (HM). This symmetry is disrupted by the integration of ports, heating and diagnostics systems. As a consequence, the cooling loops of the panels are not identical between the HM: number of branches, panel geometry and pressure drop characteristics. The results of the simulations performed for all $\mathrm{HM}$ of the panel cooling loop 3c when the orifice shown in Fig. 6 is installed in the branch with the nominal lower pressure drop are summarized in Fig.8 and 9. Fig.8 shows the flow rate in each of the panels. The maximum flow rate is $0.4 \mathrm{~kg} / \mathrm{s}$ for the panel type P08 in HM41. Fig. 9 shows the benefit of the installation of the orifice by comparing the flow rate evolution with and without orifice for each panel. The flow rate is decreased for only two panels in one HM. This flow reduction is acceptable due to the small loaded area of the panels.

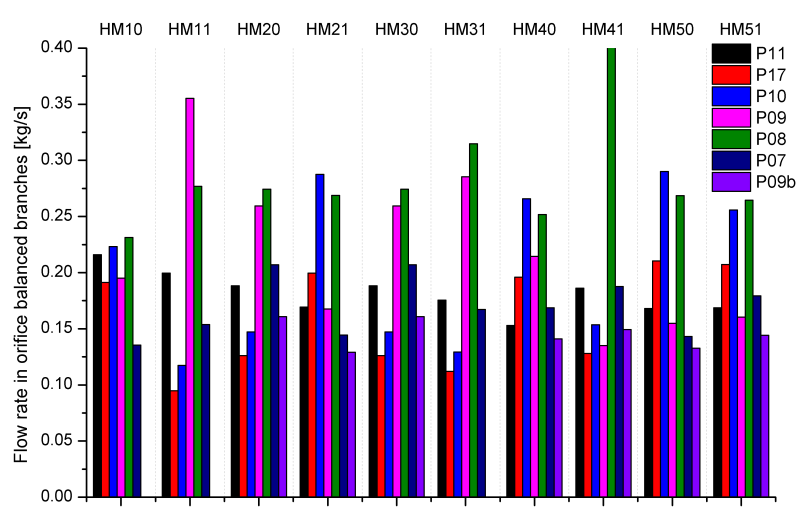

Figure 8: Flow rate increase in all panels for each half module (HM) in W7-X with orifices installed in the branches of the discharge electrode.

\section{Conclusion}

The experiments purpose to check the mechanical adjustment from the target element to the frame had 3 goals:

- Is the spring stacks tension enough to tighten the screws over cyclic mechanical load?

- Is the function of the disc spring stack as designed? Is the spring deflection in the defined range?

- Are the measured strain values in the same order of magnitude as in the simulation?

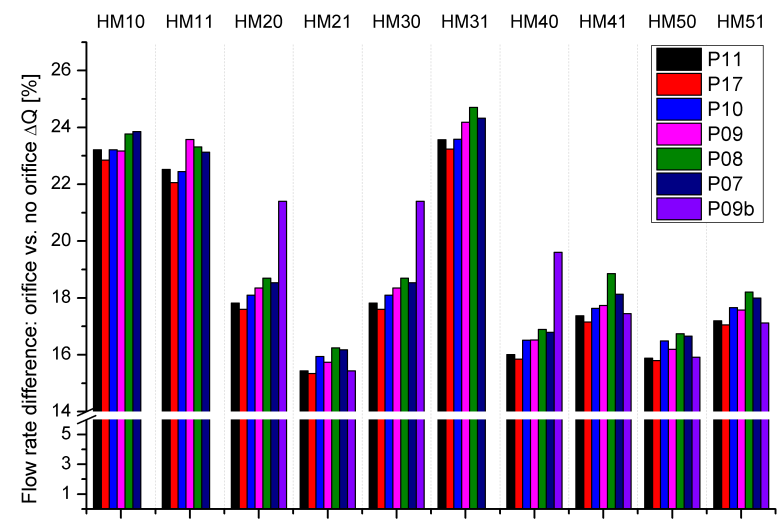

Figure 9: Flow rate increasing effect of the orifice compared to the unbalanced case.

\section{Acknowledgments}

The authors wish to thank the ITZ-Garching for its support and in particular G.Zangl for the pressure drop measurements.

[1] R. Stadler and et al. The in-vessel components of the experiment wendelstein 7-x. Fusion Engineering and Design, 84:305 - 308, 2009.

[2] J. Boscary and et al. Design and technological solutions for the plasma facing components of wendelstein 7-x. Fusion Engineering and Design, 86:572 - 575, 2011.

[3] B. Mendelevitch and et al. Design analysis and manufacturing of the cooling lines of the in vessel components of wendelstein 7-x. Fusion Engineering and Design, 86:1669 - 1672, 2011.

[4] B. Mendelevitch and et al. Lessons learned from the design and fabrication of the baffles and heat shields of wendelstein 7-x. this conference, 2012.

[5] A. Peacock and et al. The procurement and testing of the stainless steel in-vessel panels of the wendelstein 7-x stellarator. Fusion Engineering and Design, 86:1706 - 1709, 2011.

[6] D S Miller. Internal Flow System. Publisher: Miller Innovations, 1990.

[7] M. Smirnow and et al. Development of a thermo-hydraulic bypass leakage test method for the wendelstein 7-x target element cooling structure. Fusion Engineering and Design, 86:1732 1735, 2011. 